\title{
S-22: Upper Extremity Plyometric Training for the Pediatric Overhead Athletes; Randomized Controled Trial
}

\author{
Elif Turgut $^{1}$, Özge Cinar Medeni ${ }^{2}$, Filiz Fatma Çolakoğlu ${ }^{3}$, Gül Baltacı ${ }^{4}$ \\ ${ }^{1}$ Hacettepe University, Department of Physiotherapy and Rehabilitation, Ankara, Turkey \\ ${ }^{2}$ Çankırı Karatekin University, Department of Physiotherapy and Rehabilitation, Çankırı, Turkey \\ ${ }^{3}$ Gazi University, School of Sports Sciences Ankara, Turkey \\ ${ }^{4}$ Private Guven Hospital, Department of Physiotherapy and Rehabilitation Ankara, Turkey
}

\begin{abstract}
INTRODUCTION/ PURPOSE: The purpose of the current study was to investigate the effects of a 12-week upper extremity plyometric training program on upper body explosive power, strength and endurance in pediatric overhead athletes.

MATERIALS-METHOD: Twenty-eight female pediatric volleyball players participated in the study. The participants were randomly divided into two study groups: an intervention group (upper extremity plyometric training, $n=14)$ and a control group $(n=14)$. All of the participants were assessed before and after a 12-week training program for upper body explosive power, strength and endurance. Statistical comparison was performed using an analysis of variance test.

FINDINGS: Comparisons showed that after a 12-week training program, the upper body plyometric training program resulted in more improvements in an overhead medicine-ball throwing distance and a push-up performance when compared to control training.

DISCUSSION / CONCLUSION: Compared to regular training, upper body plyometric training resulted in additional improvements in upper body power and strength and endurance among female pediatric volleyball players. The findings of the study provide a basis for developing training protocols for pediatric volleyball players.
\end{abstract}

Available at: http://journalofsportsmedicine.org and http://dx.doi.org/10.5152/tjsm.2017.026

(C2017 Türkiye Spor Hekimleri Derneği. Tüm hakları saklıdır. 